\title{
Unilateral Absence of the Pulmonary Artery Incidentally Found After an Episode of Hemoptysis
}

\author{
Yoshimichi Komatsu, Masayuki Hanaoka, Michiko Ito, Masanori Yasuo, \\ Kazuhisa Urushihata, Tomonobu Koizumi, Keisaku Fujimoto and Keishi Kubo
}

\begin{abstract}
A 29-year-old woman was admitted to our hospital because of hemoptysis and dyspnea. A chest radiographic study revealed an enlargement of the right lung hilum, and the left pulmonary artery could not be clearly visualized on contrast-enhanced CT scan. Both perfusion scintigraphy and pulmonary angiography confirmed the absence of the left pulmonary artery, and agenesis of the right upper pulmonary artery. Her clinical status has been stable for the previous two years of follow-up, even though she required long-term oxygen therapy. This is a rare case of a young woman with the unilateral absence of the left pulmonary artery incidentally found after an episode of hemoptysis.
\end{abstract}

Key words: unilateral absence of pulmonary artery, pulmonary hypertension, hemoptysis

(DOI: 10.2169/internalmedicine.46.0257)

\section{Introduction}

The congenital unilateral absence of a pulmonary artery (UAPA) is a rare anomaly. It is thought to be the result of a failure in the connection of the sixth aortic arch with the pulmonary trunk during embryologic development (1). UAPA is frequently associated with abnormal lung formation and cardiovascular anomaly. We herein report a case of UAPA without any other pulmonary or cardiac anomalies, which was discovered incidentally following an episode of hemoptysis.

\section{Case Report}

A 29-year-old woman was admitted to our hospital because of hemoptysis (about $5 \mathrm{ml}$ of fresh blood) and dyspnea. She was a clerical employee and a non-smoker. Her physical development was normal, and she had no history of health problems. As a school student she had regularly taken part in physical education classes. However, for the preceding several months she felt shortness of breath for about 1530 minutes when she played tennis. None of her family members had a history of congenital cardiovascular disease.

Upon admission, her temperature was $36.4^{\circ} \mathrm{C}$, pulse rate of 64 beats/min, respiration rate of $18 / \mathrm{min}$, and blood pressure of $100 / 52 \mathrm{mmHg}$ in her bilateral arms. No clinical signs of edema, cyanosis, cervical bruits or clubbing of the fingers were found. On auscultation, the second heart sound was loud, and a diastolic murmur was present in the right parasternal portion of the fourth intercostal space. No abnormal breath sounds were heard on auscultation of the lungs. The laboratory examination showed that the inflammatory markers, such as the white blood cell count, C-reactive protein, and the erythrocyte sedimentation rate were all normal. Immunological tests showed the perinuclear pattern antineutrophil cytoplasmic antibody (p-ANCA), cytoplasmic ANCA (c-ANCA) to be negative. Data for coagulation showed the fibrinogen degradation products (FDP)-Ddimers, protein $\mathrm{C}$, protein $\mathrm{S}$, lupus anticoagulant to be normal. An arterial blood gas analysis showed the $\mathrm{pH}$ to be 7.41, with $\mathrm{PaO}_{2}$ of 63.9 Torr, $\mathrm{PaCO}_{2}$ of 36.5 Torr, $\mathrm{HCO}_{3}{ }^{-}$ of $23.1 \mathrm{mmol} / \mathrm{L}$ and $\mathrm{BE}$ of $-0.5 \mathrm{mmol} / \mathrm{L}$ on room air. Right axis deviation was observed on electrocardiogram, but no other typical right heart overload signs, such as pulmonary $\mathrm{p}$ waves or increase of $\mathrm{R}$ wave in $\mathrm{V}_{1}, \mathrm{~V}_{2}$ were detected. An echocardiogram showed severe pulmonary hypertension [Pressure Gradient (Right ventricle-Pulmonary artery) 62 $\mathrm{mmHg}$, but no apparent structural abnormality of the heart was detected. In addition, the enlargement of right heart was 


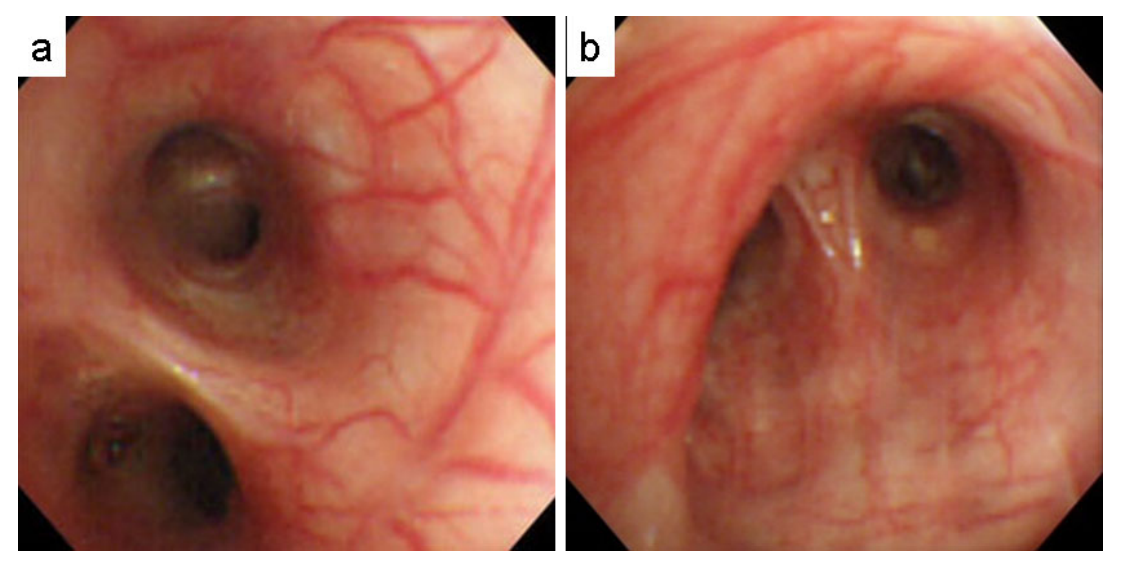

Figure 1. A bronchoscopic exam showed a highly developed plexus of mucosal vessels and vascular enlargement on all the observable bronchi in the left second carina (a), as well as at the orifice of the right upper lobe (b).

negative. The pulmonary function test showed the predicted values of vital capacity (VC) of $75.4 \%$, ratio of the forced expiratory volume in 1 second to the forced vital capacity $\left(\mathrm{FEV}_{1} / \mathrm{FVC}\right)$ of $90.2 \%$, and diffusion capacity for carbon monoxide $\left(\mathrm{DL}_{\mathrm{co}}\right)$ of $59.0 \%$, indicating a restrictive ventilatory impairment and a diffusing capacity abnormality. The bronchoscopic examination revealed a highly developed plexus of mucosal vessels and vascular enlargement of all of the observable bronchi in the left lung (Fig. 1a), as well as at the orifice of the right upper lobe (Fig. 1b).

On imaging examinations, the posterior-anterior radiographic view of the chest revealed an enlargement of the right lung hilum and an increased vascularity in the right lower lung field (Fig. 2). A computed tomographic study (CT) of the chest demonstrated a consolidation, which was surrounded by a ground glass opacity in the right S3 segment (Fig. 3a), suggesting an aspiration of hemorrhage at the time of the hemoptysis. A contrast-enhanced CT scan showed an enlargement of the pulmonary artery trunk, and the left pulmonary artery was visible because the blood flow might have been fed by the bronchial artery (Fig. 3b). Furthermore, the magnetic resonance angiographic study (MRA), coronal section, demonstrated the absence of the left pulmonary artery, and poor perfusion in the right apical region (Fig. 4). Similarly, the ventilation scintigraphy (Xe gas) showed an equal distribution in both lung fields (Fig. 5a), but the perfusion scintigraphy (Tc-MAA) revealed an absence of perfusion in the left lung and poor perfusion in the apical region of the right lung (Fig. 5b).

We strongly suspected a congenital unilateral absence of the left pulmonary artery in this case, according to the radiologic findings. Therefore, a pulmonary arteriographic study was performed, which confirmed the absence of the left pulmonary artery (Fig. 6b), and agenesis of the right upper pulmonary artery, which was compensated for by an enlarged right descending pulmonary artery (Fig. 6a).

We performed cardiac catheterization on room air, and the pulmonary artery pressure was 66/18/38 (systolic/diastolic/ mean) $\mathrm{mmHg}$. When administering oxygen at $2 \mathrm{~L} / \mathrm{min}$ and

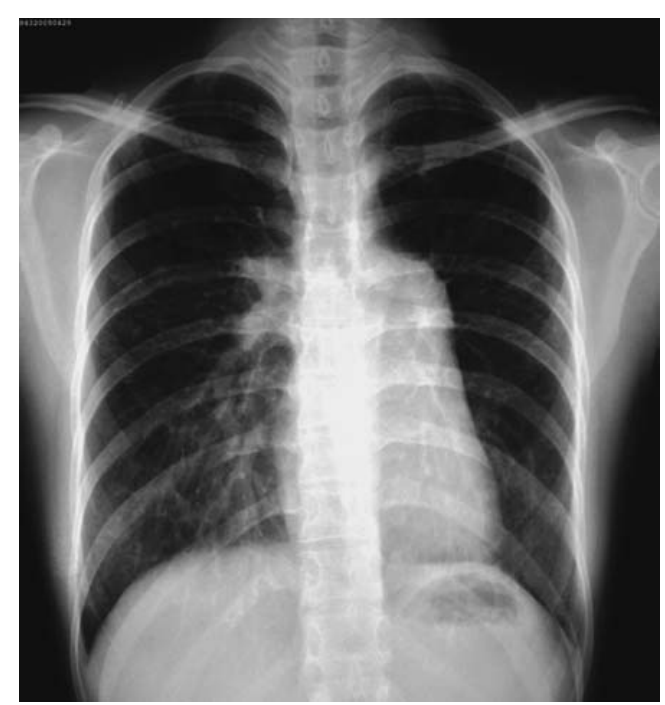

Figure 2. The posterior-anterior view of chest radiograph showed an enlargement of the right lung hilum and an increased vascularity in the right lower lung field.

$10 \mathrm{~L} / \mathrm{min}$, the pulmonary artery pressure improved to 55/14/ $28 \mathrm{mmHg}$ and 48/14/28 $\mathrm{mmHg}$, respectively. Therefore, long-term oxygen therapy at $2 \mathrm{~L} / \mathrm{min}$ was prescribed as treatment. Her clinical status has remained stable for the past two years of follow-upon an outpatient basis, with no recurrence of hemoptysis.

\section{Discussion}

In 1868, UAPA was first described as a rare developmental anomaly (2). UAPA is also referred to as proximal interruption of the pulmonary artery, because in some cases surgical dissection revealed an incompletely developed pulmonary artery of the hilum (3). There is no predilection for the right or left side, although the condition is somewhat more common on the right. However, left-sided agenesis seems to be more frequently associated with cardiac abnormalities (4). We herein report a rare case of UAPA in a patient with 

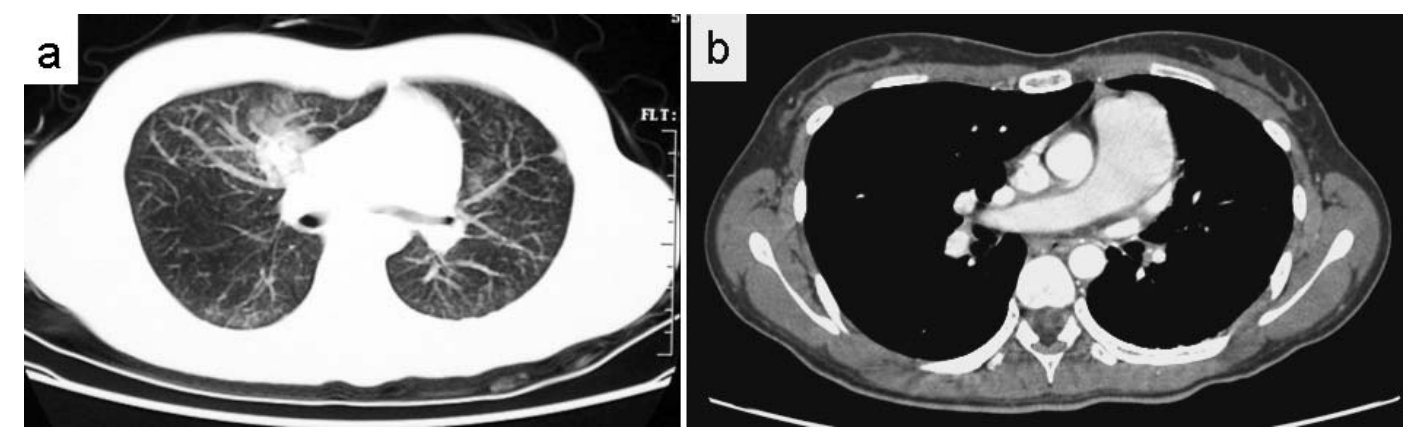

Figure 3. a: Chest computed tomography (CT) demonstrated a consolidation that was surrounded with ground glass opacity in the right S3 segment. b: The contrast-enhanced CT scan showed an enlargement of the pulmonary artery trunk, and the left pulmonary artery was visible because the blood flow was likely fed by the bronchial artery.

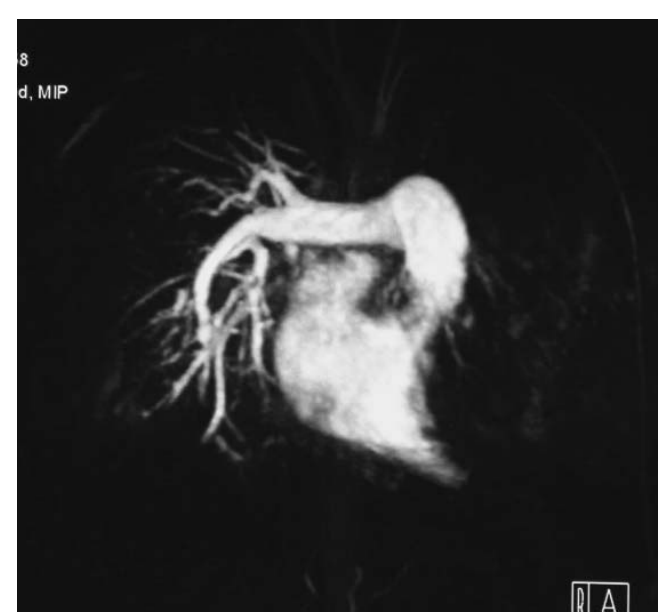

Figure 4. Magnetic resonance angiography in a coronal section revealed the absence of the left pulmonary artery, and a poor image in the right apical lung.

absence of the left pulmonary artery without associated cardiac malformations. Moreover, her right upper pulmonary artery was also incompletely developed.

The diagnosis of UAPA is generally based on medical history, physical examination findings, and the results of chest radiographs. A case of UAPA shows a characteristic pattern of chest radiographic findings, including cardiac and mediastinal displacement, absence of the pulmonary artery shadow, smaller hemithorax, elevation of the diaphragm, and a paucity of the vascular markings on the involved side (57). To compensate for the absence, there may be hyperinflation and herniation of the unaffected lung across the midline. In the present case, volume loss of the lung on the affected side and hyperinflation of the lung on the healthy side were not found. The absence of hyperinflation or collapse of either lung, or cardiac anomaly is probably the reason for the negative findings in her previous health screenings. Therefore, in our case the CT scan, MRI scan, ventilation/perfusion scan, and finally the angiography findings were absolutely necessary in order to establish an accurate diagnosis. The current case with UAPA was differentiated from vasculitis syndrome, aortitis syndrome and pulmonary thrombolism based on the clinical manifestations and the findings of laboratory examinations which showed normal ANCA related markers, inflammatory reaction and coagulation system factor (FDP-D-dimer). In addition, contrastenhanced CT showed no thrombus.

Previous reports have stated that $30 \%$ of patients with UAPA can remain asymptomatic for a long period of time $(5,6)$. Some patients may present with recurrent infections, dyspnea on exertion, or congestive heart failure $(5,6)$. Pulmonary hypertension was found in $44 \%$ of the patients (6). Hemoptysis occurs in up to $10 \%$ of patients, secondary to either hypertrophied bronchial collateral vessels or the presence of a peripheral arteriovenous fistula $(5,6,8)$. Fiberoptic bronchoscopic examination may reveal plexuses of dilated blood vessels, mesh-like vascularization, and erythema in the affected side bronchi, which can cause hemoptysis (9). The unaffected side does not usually exhibit any such vascular changes (10). In the case presented, the bronchoscopic examination showed a highly developed plexus of mucosal vessels and vascularization, not only in the left lung, but also in the orifice of the right upper lobe. As a result, these mucosal vascular abnormalities could be the source of the bleeding which resulted in hemoptysis in this patient, as supported by the findings of the CT scan.

Regarding the treatment of UAPA, it is reported that $8 \%$ of the patients underwent either a pneumonectomy or lobectomy for recurrent hemoptysis or intractable pulmonary infections (6), and $7 \%$ of the patients underwent revascularization of hidden pulmonary arteries (6). When pulmonary hypertension is present, revascularization of the absent artery is recommended in order to improve the hypoxic condition (6). For this case, we decided to administer oxygen therapy because of the improvement of pulmonary hypertension noted during cardiac catheterization, when oxygen was administered.

Respiratory symptoms, such as breathlessness, hemoptysis, and pulmonary hypertension may also occur in patients at elevations of 1,800 to 2,100 meters, and fatal cases have been reported at these altitudes (11). Pulmonary hyper- 


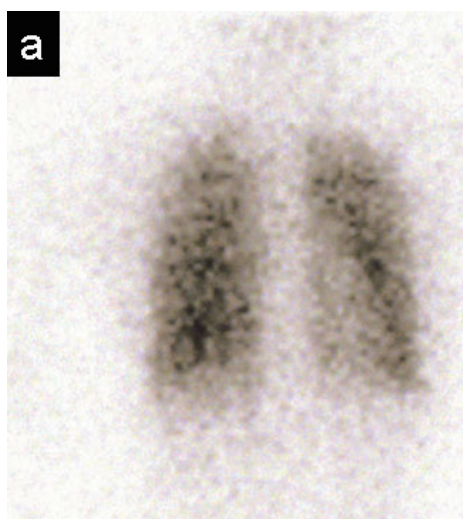

b

Figure 5. a: The ventilation scintigraphy (Xe gas) showed equal distribution in both lungs. b: The perfusion scintigraphy (Tc-MAA) revealed absence of perfusion in the left lung (arrow).

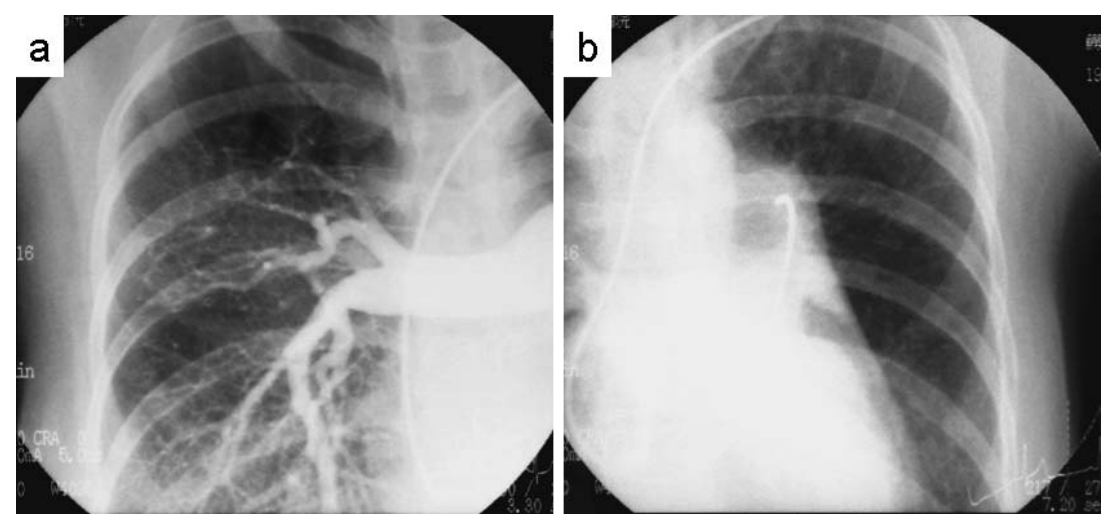

Figure 6. The pulmonary arteriography confirmed the absence of the left pulmonary artery (b), and an agenesis of the right upper pulmonary artery compensated by an enlarged right descending pulmonary artery (a).

tension may cause distress, especially when it develops during pregnancy. In a report regarding three UAPA patients with pulmonary hypertension, one of the patients died during pregnancy (6). The overall mortality rate of UAPA was reported to be $7 \%$ (6). We explained the risk factors regarding the absence of a pulmonary artery to our patient, including pregnancy, hard physical labor, and travel to a high alti- tude. She returned to her work while receiving long-term oxygen therapy, and her clinical status has remained stable, without any recurrence of hemoptysis, during the follow-up on an outpatient basis for the past two years. It is necessary for this patient to be followed up closely, especially for the observation of her pulmonary hemodynamics.

\section{References}

1. Cucci CE, Doyle EF, Lewis EW Jr. Absence of a primary division of the pulmonary trunk. An ontogenetic theory. Circulation 29: 124-131, 1964.

2. Frantzel O. Angeborener Defect der Rechten Lungenarterie. Virchows Arch Pathol Anat 43: 420, 1868 (in German).

3. Canver CC. Isolated unilateral pulmonary artery agenesis (letter). Thorac Cardiovasc Surg 105: 766, 1993.

4. Vergauwen S, Bracke P, De Schepper A. Unilateral absence of a pulmonary artery. J Belge Radiol 81: 254, 1998.

5. Bouros D, Pare P, Panagou P, Tsintris K, Siafakas N. The varied manifestation of pulmonary artery agenesis in adulthood. Chest 108: 670-676, 1995.

6. Harkel DJT, Bloom NA, Ottenkamp J. Isolated unilateral absence of a pulmonary artery. A case report and review of the literature. Chest 122: 1471-1477, 2002.

7. Debatin J, Moon R, Spritzer C, Macfall J, Sostman H. MRI of ab- sent left pulmonary artery. J Comput Assist Tomogr 16: 641-645, 1992.

8. Lip GY, Dunn FG. Unilateral pulmonary artery agenesis: a rare cause of hemoptysis and pleuritic chest pain. Int J Cardiol 40: 121-125, 1993

9. Matsushima H, Matsuura K, Kohiyama R, Kobayashi Y, Tanaka O, Kanazawa M. Recurrent hemoptysis as the right pulmonary artery. Jpn J Chest Dis 59: 287-292, 2000 (in Japanese).

10. Mimura S, Kobayashi H, Shinkai M, Kanoh S, Motoyoshi K. A case report of congenital isolated absence of the right pulmonary artery: bronchofibroscopic findings and chest radiological tracings over 9 years. Respirology 10: 250-253, 2005.

11. Hackett PH, Creagh CE, Grover RF, et al. High-altitude pulmonary edema in persons without the right pulmonary artery. N Engl Med 302: 1070-1073, 1980.

(C) 2007 The Japanese Society of Internal Medicine http://www.naika.or.jp/imindex.html 